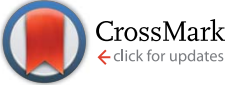

Cite this: RSC Adv., 2014, 4, 31594
Received 30th April 2014 Accepted 15th July 2014

DOI: 10.1039/c4ra03961k

www.rsc.org/advances

\title{
A new class of pyrenyl solid-state emitters: 1-pyrenyl ynones. Synthesis via the Friedel-Crafts route, molecular and electronic structure and photophysical properties $\uparrow$
}

\author{
Rafał Flamholc, ${ }^{a}$ Damian Plażuk, ${ }^{a}$ Janusz Zakrzewski, ${ }^{\star a}$ Rémi Métivier, ${ }^{\star b}$ \\ Keitaro Nakatani, ${ }^{b}$ Anna Makal ${ }^{c}$ and Krzysztof Woźniak ${ }^{c}$
}

\begin{abstract}
Friedel-Crafts acylation of pyrene with alkynoic acids in the presence of trifluoroacetic anhydride and triflic acid constitutes a direct and efficient route to 1-pyrenyl ynones. These compounds in chloroform solution emit fluorescence at longer wavelengths, with higher quantum yields and longer lifetimes than a typical saturated acylpyrene derivative, 1-acetylpyrene. Moreover, in contrast to 1-acetylpyrene, they are moderate solid-state emitters. Comparative DFT studies revealed strong stabilization of the LUMOs of 1pyrenyl ynones in comparison to the LUMO of 1-acetylpyrene. The single-crystal X-ray structure of 1(pyren-1-yl)but-2-yn-1-one showed $\pi$-interactions of pyrenyl moieties in the crystal lattice. Investigations of the solid-state fluorescence of this compound revealed emission from long-lived excited states, including excimer species.
\end{abstract}

\section{Introduction}

Pyrene and its derivatives have attracted considerable interest as materials for organic electronics, ${ }^{1}$ fluorescent monomers, ${ }^{2,3}$ molecular probes, ${ }^{4-7}$ and sensors. ${ }^{\mathbf{8} 9}$ Considerable effort has been focused on the development of synthetic routes to pyrenes bearing different substituents $\mathbf{s}^{\mathbf{1 , 1 0 - 1 2}}$ and on studies of their fluorescence properties. ${ }^{\mathbf{1 3}, 14}$ Special attention has been paid to 1pyrenyl carbonyl compounds such as aldehyde, ketones, acids, esters, amides, etc., exhibiting strongly environment-sensitive fluorescence and used as molecular probes. ${ }^{15-21}$ Recent works by the Konishi group have provided a basis for understanding the photophysical properties of this class of fluorophores. ${ }^{15-17,21}$ As far as pyrenyl ketones are concerned, research reports have mainly concentrated on pyrenyl alkyl ketones. This class of perylenyl fluorophores shows short fluorescence lifetimes $\left(10^{-9}-10^{-8} \mathrm{~s}\right.$, compared with $>10^{-7} \mathrm{~s}$ for pyrene) and low quantum yields due to efficient intersystem crossing. ${ }^{14,15}$ Only very recently have two reports appeared disclosing the synthesis

${ }^{a}$ Department of Organic Chemistry, Faculty of Chemistry, University of Łódź, Tamka 12, 91-403 Eódź, Poland. E-mail: janzak@uni.lodz.pl

${ }^{b} P P S M$, ENS Cachan, CNRS, UniverSud, 61 av President Wilson, 94230 Cachan, France.E-mail: metivier@ppsm.ens-cachan.fr

${ }^{c}$ Department of Chemistry, Warsaw University, Pasteura 1, 02-093 Warszawa, Poland $\dagger$ Electronic supplementary information (ESI) available: Syntheses of 1a-d. Electronic absorption and emission spectra of $\mathbf{1 a - d}$ in various solvents. Details of X-ray diffraction and photophysical studies. CCDC 996918. For ESI and crystallographic data in CIF or other electronic format see DOI: 10.1039/c4ra03961k and photophysical properties of the simplest pyrenyl alkynyl ketone, 1-propynoilpyrene. ${ }^{22,23}$ This ketone offers a unique opportunity for attachment of a pyrene carbonyl tag to biomolecules or nanomaterials via azide-alkyne click chemistry.

In a continuation of our research programme, which has focused on the use of functionalised carboxylic acids as acylating agents in Friedel-Crafts reaction, we recently elaborated an efficient method of synthesis of ferrocenyl ynones via a direct reaction of ferrocene with alkynoic acids in the presence of trifluoroacetic anhydride (TFAA) and trifluoromethanesulfonic acid (TfOH) ${ }^{24}$ Herein we report that this approach may be used for simple and efficient synthesis of 1-pyrenyl ynones. We have also studied the fluorescence properties of these compounds which were compared to those of the simplest pyrenyl alkyl ketone, 1-acetylpyrene. Unexpectedly, we found that, in contrast to the latter compound, the synthesized 1-pyrenyl ynones display fluorescence not only in solution but also in the solid state. An X-ray diffraction study (including topological analysis of experimental charge density) performed for 1-(pyren-1-yl)but2 -yn-1-one revealed face-to-face $\pi$-stacking of the pyrene moieties in the crystal, thus suggesting that emission may originate from solid-state excimers. Finally, we carried out comparative DFT calculations on this compound and 1-acetylpyrene.

\section{Results and discussion}

\section{Synthesis of pyrenyl ynones 1a-d}

Ynones or $\alpha, \beta$-acetylenic ketones are versatile building blocks in organic synthesis, especially in the synthesis of heterocyclic 
compounds. ${ }^{25}$ They can be synthesized via various routes starting from terminal alkynes. ${ }^{26-29}$ Readily available 2-alkynoic acids $^{\mathbf{3 0}}$ are another potential starting material in ynone synthesis via Friedel-Crafts acylation of arenes. We reported the first example of such reaction, in which ferrocene was used as a reactive $\pi$-rich arene. ${ }^{24}$ Since pyrene also exhibits high reactivity towards electrophiles, it seemed interesting to evaluate whether this approach can be used for a direct introduction of the alkynoyl group into this polycyclic arene.

The Friedel-Crafts reaction of pyrene with 2-alkynoic acids (Scheme 1) was carried out under conditions described earlier for ferrocene. ${ }^{24}$ The isolated yields of compounds $1 \mathbf{1 a}-\mathbf{c}$ were in the range of $69-74 \%$. Similarly, as in the case of ferrocene, the reaction of pyrene with propiolic acid $(\mathrm{R}=\mathrm{H})$ led to an intractable reaction mixture. However, compound 1d was prepared in an almost quantitative yield (99\%) by fluoride-promoted desilylation of 1c.

Structures of synthesized ynones 1a-d were confirmed by spectroscopic and elemental analysis data (see ESI $\dagger$ ). The simplest compound of this series, 1d, was already reported in the literature. It was synthesised $40-44 \%$ overall in a reaction of pyrene-1-carboxaldehyde with ethynyl magnesium bromide ${ }^{22}$ (or TMS-ethynyl magnesium bromide followed by desilyla$\operatorname{tion}^{23}$ ), and subsequent oxidation of the alcohol formed with Jones reagent. Compared to those methods our synthesis was simpler (using pyrene as the starting material) and more efficient $(71 \%$ overall yield).

\section{Molecular structure and crystal packing of 1a}

Crystals of 1a that were suitable for X-ray diffraction study were obtained by slow diffusion of $n$-heptane into a solution of this compound in chloroform. Under these conditions 1a crystallized in the centrosymmetric $P 2_{1} / c$ space group in a monoclinic system, with one independent molecule in the crystallographic asymmetric unit located in general position. The molecular structure of 1a is presented in Fig. 1.

In the experimental structure of 1a the pyrenyl moiety is not ideally planar, but slightly bent along its longer $(\mathrm{C} 7 \rightarrow \mathrm{C} 14)$ axis. The angle between the plane of ring $\mathrm{C} 5, \mathrm{C} 6, \mathrm{C} 7, \mathrm{C} 8, \mathrm{C} 9$, and $\mathrm{C} 16$ (ring 1) and that of ring C1, C2, C12, C13, C14, C15 (ring 2)

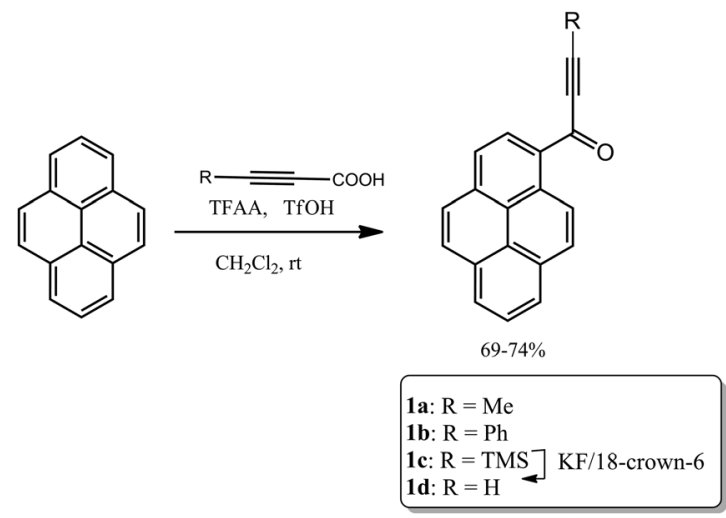

Scheme 1 Synthesis of 1-pyrenyl ynones 1a-d.

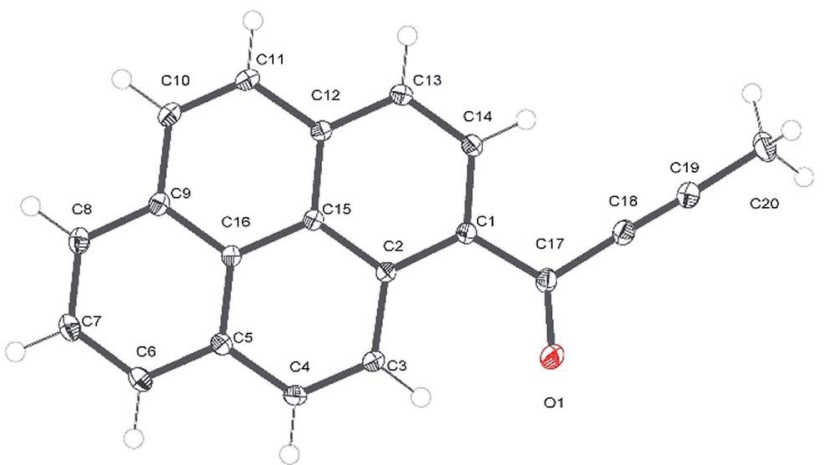

Fig. 1 ORTEP representation of 1 a with an atom numbering scheme. Atomic displacement parameters are drawn at 50\% probability level.

is 3.7(3) degrees. The propynoyl substituent is twisted out of the plane of adjacent ring 2, as indicated by the $\mathrm{C} 2-\mathrm{C} 1-\mathrm{C} 17-\mathrm{C} 18$ and C14-C1-C17-O1 torsion angles which were significantly different from $180^{\circ}$ (172.37(7) degrees and C14 C1 C17 O1 168.68(9) degrees, respectively). The angle between the plane of ring 2 and the plane of the carbonyl moiety was 9.9(5) degrees. This conformation enables the formation of two weak intermolecular $\mathrm{C}-\mathrm{H} \cdots \mathrm{O}$ hydrogen bonds in the crystal lattice: $\mathrm{C} 4-$

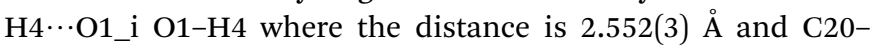

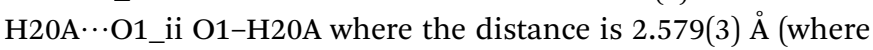
(i) denotes the following symmetry transformation: $1-x, 2-y$, $1-z$ and (ii) denotes the symmetry transformation: $x, 11 / 2-y$, $1 / 2+z$ ) (Fig. 2).

The tilt of the carbonyl group out of the ring 2 plane does not prevent the formation of a weak intramolecular $\mathrm{C} 3-\mathrm{H} 3 \cdots \mathrm{O} 1$ hydrogen bond, with an $\mathrm{O} 1 \cdots \mathrm{H} 3$ distance of only $2.156(1) \AA$. Viewed along the crystallographic [001] direction, the crystal packing of 1a shows a characteristic herringbone motif (Fig. 3a) with distinct layers of molecules stacked along the [100] direction. Within the layers the carbonyl moieties are oriented almost parallel to the [001] direction; in consecutive layers the carbonyl groups point in opposite directions (i.e. the carbonyl moieties in one layer are almost parallel to [001] direction, while the carbonyl moieties from the next layer are almost antiparallel

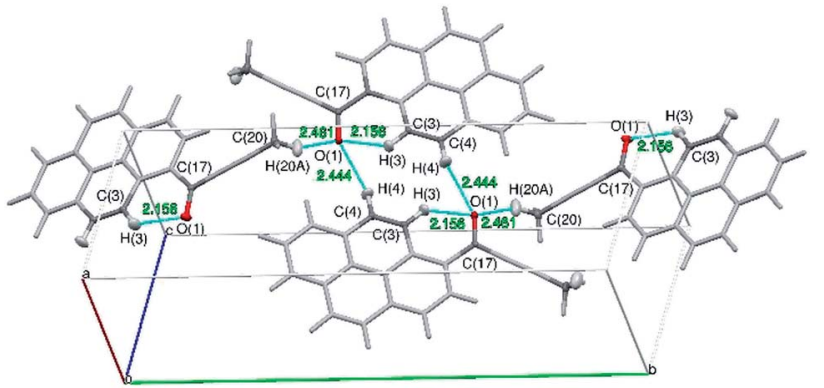

Fig. $2 \mathrm{C}-\mathrm{H} \cdots \mathrm{O}$ hydrogen bonds in a crystal structure of $1 \mathrm{a}$. The atoms involved in the hydrogen bonds are presented with thermal ellipsoids at $50 \%$, the remaining atoms are represented in grey for clarity. The hydrogen bonds are highlighted in cyan. The $\mathrm{H} \cdots \mathrm{A}$ distances are reported in $\AA$. 
a

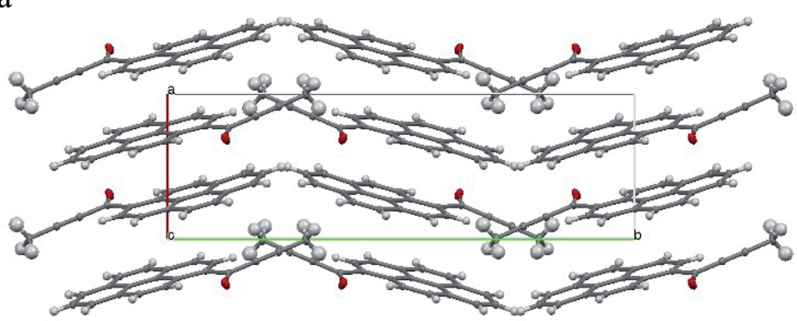

b

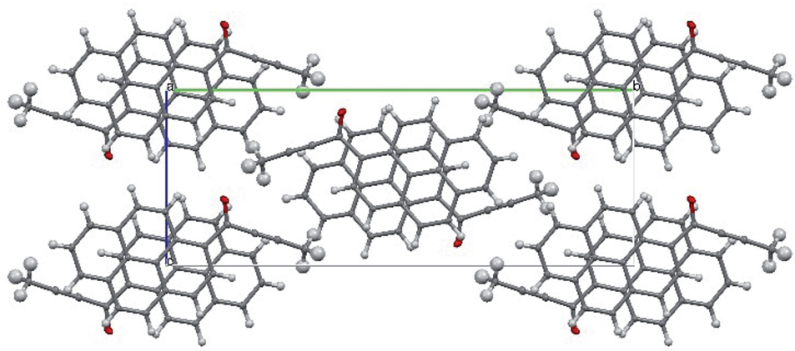

C

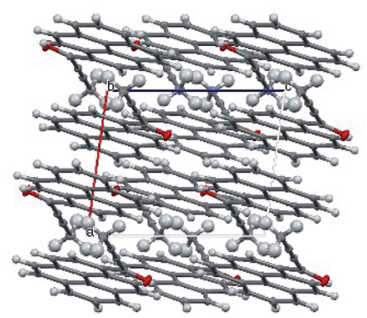

Fig. 3 Crystal packing of 1a. (a) view along the [001] direction, (b) view along the [100] direction, (c) view along the [010] direction. Crystal axes denoted as follows: a [100] as red, b [010] as green and c [001] as blue.

to this direction). The angle between the $\mathrm{C} 17-\mathrm{O} 1$ carbonyl bond and the crystallographic [001] direction is 25.8(5) degrees (for a molecule in the alternative layer this would be 205.8(5) degrees).

The crystal structure of 1a can be considered as composed of layers of molecules with parallel dipole and transition moments; with the directions of these moments alternating between layers. Each layer can be further viewed as composed of strands of molecules, packed along the [001] direction.

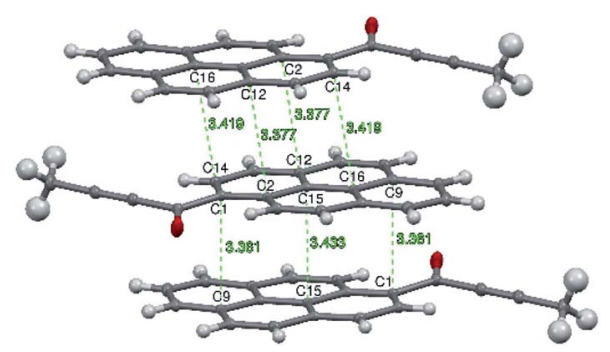

Fig. 4 Intermolecular contacts in layers of 1a molecules along the [100] direction. The C...C distances are highlighted in green and reported in A. Atomic displacement parameters are reported at 50\% probability level.
Molecules from consecutive layers along the [100] direction (Fig. 3b), related by crystallographic centres of inversion, are also involved in $\pi$-stacking interactions, thus building columns of symmetry-related molecules along [100] (Fig. 3a). Due to the 'bend' in the pyrene moiety, the closest contacts of an independent molecule (I) and the symmetry-related molecule II (above) are different from the closest contacts of an independent molecule (I) and the symmetry-related molecule III (below) (Fig. 4). For the former the closest contacts are C2_I-C12_II

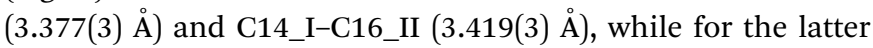
the closest contacts are C1_I-C9_III (3.361(3) ̊̊) and C15_IC15_III (3.433(3) ̊̊).

Interlayer interactions are stabilised by a weak $\mathrm{C}-\mathrm{H} \cdots \mathrm{O}$ hydrogen bond: $\mathrm{C} 4-\mathrm{H} 4 \cdots \mathrm{O} 1 \_\mathrm{i}$, while the separate layers are stabilised by a weak $\mathrm{C}-\mathrm{H} \cdots \mathrm{O}$ hydrogen bond: $\mathrm{C} 20-\mathrm{H} 20 \mathrm{~A} \cdots \mathrm{O} 1$.

Because the scattering power of the 1a crystals was good, aspherical atomic scattering factors could be applied in structure refinement. Therefore, we could also perform a preliminary topological analysis of experimental charge density for this compound (see ESI†). It supports the structural analysis, thus confirming the presence and significance of all of the already mentioned $\mathrm{C}-\mathrm{H} \cdots \mathrm{O}$ interactions. The strongest hydrogen bond that is present in the crystals of $1 \mathrm{a}$ is the intramolecular C3$\mathrm{H} 3 \cdots \mathrm{O} 1$ bond, according to electron density and energy density criteria. A ring critical point was found within the $\mathrm{C} 1-\mathrm{C} 2-\mathrm{C} 3-$ H3-O1-C17 ring, closed by this bond, thus confirming the significance of this interaction according to Koch and Popelier criteria. ${ }^{31}$ This analysis also demonstrated bond paths for the $\pi$ stacking interactions between symmetry-related molecules of 1a, which may support the 'excimer' hypothesis (vide infra). (Table 1)

The molecule of $1 \mathrm{a}$ in the crystal is strongly polarized with a negative charge of over $0.5 \mathrm{e}$ localised on the butynoyl group and an equivalent positive charge residing on the pyrene moiety. This results in a significant molecular dipole moment, oriented in between the $\mathrm{C} 1-\mathrm{C} 17$ and $\mathrm{C} 17-\mathrm{O} 1$ bond axes and coplanar with the pyrene moiety. According to the DFT B3PW91 calculations, an isolated molecule of 1a has the dipole moment of $3.8 \mathrm{D}$, while the dipole moment obtained from the experimental charge density model is almost 4 times larger $(15.8 \mathrm{D})$. It must be stressed that the absolute value of the molecular dipole moment in the crystal cannot be reliably derived from the current experimental charge density model, and that the value only indicates a tendency for increased polarisation of the molecule in the crystalline environment. Quantum chemical calculations in the periodic lattice performed at the B3LYP level of theory with a $6-31+g(d)$ basis set yielded an optimised geometry that was identical to the geometry from X-ray diffraction within an experimental error. These calculations confirm enhancement of the molecular dipole moment in the crystal lattice. The resulting dipole moment has a direction that is in agreement with the results of experimental charge density analysis and the results of theoretical calculations for an isolated molecule and a value of 4.7 D. The dipole moment vector is almost perpendicular to the [100] crystallographic direction $\left(\sim 86^{\circ}\right)$ and coplanar with the pyrene moiety. 
Table 1 The most important parameters of experimental charge density at bond critical points in 1a. Symmetry operations: (i): $1-x, 2-y, 1-z$; (ii): $x, 11 / 2-y, 1 / 2+z$; (iii): $-x, 2-y,-z$; (iv): $1-x, 2-y,-z$

\begin{tabular}{|c|c|c|c|c|c|c|c|c|}
\hline Interaction & $\begin{array}{l}\rho\left(r_{\mathrm{BCP}}\right) \\
{\left[\mathrm{e}^{-3}\right]}\end{array}$ & $\begin{array}{l}\operatorname{Lap}\left(r_{\mathrm{BCP}}\right) \\
{\left[\mathrm{e}^{-5}\right]}\end{array}$ & $\begin{array}{l}G\left(r_{\mathrm{BCP}}\right) \\
{\left[\mathrm{H} \mathrm{a}_{0}^{-3}\right]}\end{array}$ & $\begin{array}{l}V\left(r_{\mathrm{BCP}}\right) \\
{\left[\mathrm{H} \mathrm{a}_{0}^{-3}\right]}\end{array}$ & $\begin{array}{l}H\left(r_{\mathrm{BCP}}\right) \\
{\left[\mathrm{H} \mathrm{a}_{0}^{-3}\right]}\end{array}$ & $\begin{array}{l}G\left(r_{\mathrm{BCP}}\right) / \rho\left(r_{\mathrm{BCP}}\right) \\
{\left[\mathrm{H} \mathrm{e}^{-1}\right]}\end{array}$ & $\begin{array}{l}H\left(r_{\mathrm{BCP}}\right) / \rho\left(r_{\mathrm{BCP}}\right) \\
{\left[\mathrm{H} \mathrm{e}^{-1}\right]}\end{array}$ & $\begin{array}{l}\left|V\left(r_{\mathrm{BCP}}\right)\right| / G \\
\left(r_{\mathrm{BCP}}\right)\end{array}$ \\
\hline $\mathrm{O} 1 \cdots \mathrm{H} 3$ & $0.110(5)$ & $2.076(4)$ & 0.017 & -0.013 & 0.004 & 1.043 & 0.245 & 0.765 \\
\hline H20A‥ii_O1 & $0.045(4)$ & $0.745(3)$ & 0.006 & -0.004 & 0.002 & 0.900 & 0.300 & 0.667 \\
\hline C2 $\cdots$ iii_C12 & $0.031(1)$ & 0.295 (1) & 0.002 & -0.002 & 0.001 & 0.435 & 0.218 & 1.000 \\
\hline C14‥iii_C16 & $0.029(1)$ & $0.272(1)$ & 0.002 & -0.002 & 0.001 & 0.465 & 0.233 & 1.000 \\
\hline
\end{tabular}

\section{Photophysical properties of 1a-d and AcPyr in solution}

The electronic absorption and emission spectra of 1a-d and AcPyr in chloroform $\left(c=10^{-6} \mathrm{M}\right)$ are shown in Fig. 5 and the spectral data are collected in Table 2. At such a low

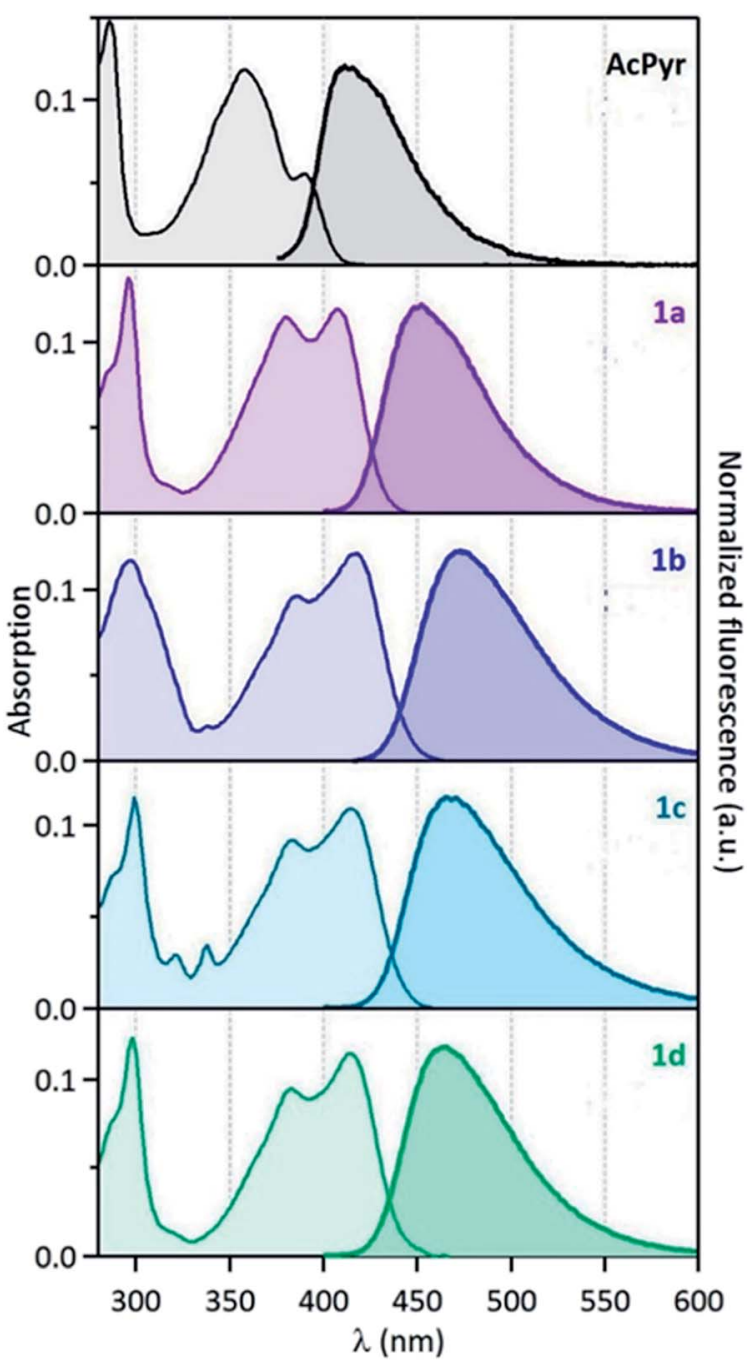

Fig. 5 Normalised absorption (left) and fluorescence (right) spectra of compounds $1 \mathrm{a}-\mathrm{d}$ and $\mathrm{AcPyr}$ in $\mathrm{CHCl}_{3}\left(\lambda_{\text {exc }}=390 \mathrm{~nm}\right.$ for $1 \mathrm{a}-\mathrm{d}$ and 330 $\mathrm{nm}$ for AcPyr). concentration the formation of aggregates and excimers is highly unlikely and the observed emission can be ascribed to fluorophore monomers.

The spectra reveal significant (up to $\sim 60 \mathrm{~nm}$ ) bathochromic shifts of the bands of 1a-d in comparison to those of AcPyr. This suggests that the alkynoyl substituents are more efficiently conjugated with the pyrenyl moiety than the acetyl group. Furthermore, the absorption bands of 1a-d enter the visible region and these compounds can be excited with violet light. All of the investigated ketones are practically nonfluorescent in a nonpolar solvent, hexane (see ESI†). In a medium polarity solvent, chloroform, compounds 1a-d showed fluorescence at 449-471 $\mathrm{nm}$ with quantum yields in the range of 0.02-0.07, whereas AcPyr emitted weakly at 409 $\mathrm{nm}$ (quantum yield was lower than 0.005). In a more polar aprotic solvent, DMSO, all 1a-d were emissive, whereas in a polar but hydroxylic solvent, methanol, only 1a was a strong emitter. Surprisingly, 1a-d were stronger emitters in chloroform than in methanol, whereas the opposite effect was observed for AcPyr. Time-resolved fluorescence investigations of 1a-d and AcPyr were performed in chloroform solutions and revealed multiexponential decays in all cases (Fig. 6a, Table 2). A fast decay was observed for AcPyr, intermediate behavior was observed for 1a and 1c, whereas much slower decays were recorded for $\mathbf{1 b}$ and $\mathbf{1 d}$. Three sets of decay time constants could be distinguished: a fast contribution $\left(\tau_{1}<0.15 \mathrm{~ns}\right)$, an intermediate contribution $\left(\tau_{2}=0.2-0.3 \mathrm{~ns}\right)$, and a slow contribution $\left(\tau_{3}>0.5 \mathrm{~ns}\right)$.

These three contributions may be related to three populations of conformers having different relaxation times and different emission efficiencies. The fraction of intensity related to the slowest component $\tau_{3}$ is very much variable depending on the compound. It is absent for AcPyr, represents a small proportion for 1a $\left(\phi_{3}=0.15\right)$ and its contribution becomes predominant for compounds $1 \mathbf{b}\left(\phi_{3}=0.80\right), 1 \mathrm{c}\left(\phi_{3}=0.59\right)$, and 1d $\left(\phi_{3}=0.88\right)$. This fraction of intensity is well-correlated to the overall fluorescence quantum yields of compounds AcPyr and 1a-d (Fig. 6b). Therefore, we could conclude that the slowest decay time corresponds to a conformer which is much more fluorescent than the others. Consequently, the fluorescence quantum yields measured in $\mathrm{CHCl}_{3}$ reflect the various proportions of the different conformers, which are variable from one compound to another. 
Table 2 Electronic absorption and fluorescence data for compounds $1 \mathrm{a}-\mathrm{d}$ and AcPyr

\begin{tabular}{|c|c|c|c|c|c|}
\hline & $1 \mathrm{a}$ & $1 b$ & $1 c$ & 1d & AcPyr \\
\hline$\lambda_{\mathrm{abs}}(\mathrm{nm})\left(\mathrm{CHCl}_{3}\right)$ & 408 & 418 & 416 & 415 & 391 \\
\hline$\varepsilon_{\max }\left(\mathbf{M}^{-1} \mathrm{~cm}\right)\left(\mathrm{CHCl}_{3}\right)$ & 15100 & 45500 & 18800 & 24800 & 15400 \\
\hline$\Phi_{\mathrm{F}}\left(\mathrm{CHCl}_{3}\right)$ & 0.02 & 0.06 & 0.05 & 0.07 & $<0.005$ \\
\hline$\lambda_{\mathrm{em}}(\mathrm{nm})($ solid$)$ & 569 & 530 & 550 & 565 & 486 \\
\hline$\Phi_{\mathrm{F}}($ solid $)$ & 0.13 & 0.04 & 0.12 & 0.07 & - \\
\hline
\end{tabular}

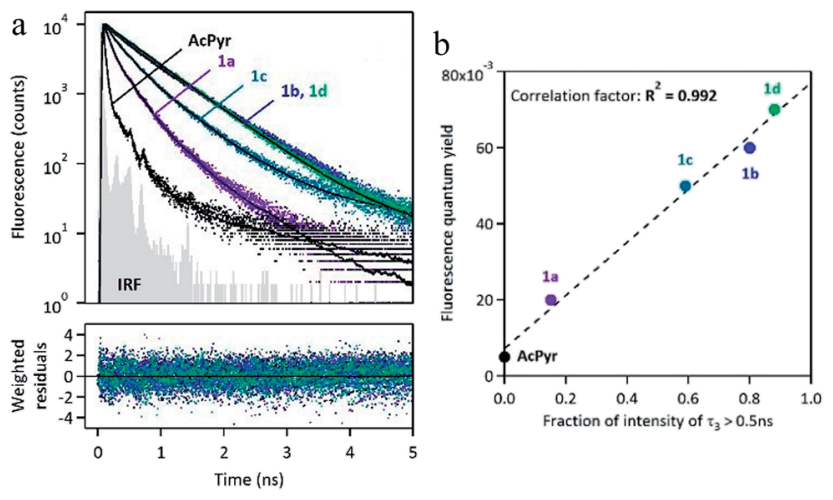

Fig. 6 (a) Fluorescence decay curves of $1 a-d$ and AcPyr recorded in $\mathrm{CHCl}_{3}$, with multiexponential fitting and weighted residuals (IRF = instrumental response function). (b) Correlation plot between the fluorescence quantum yield measured in $\mathrm{CHCl}_{3}$ and the fraction of intensity corresponding to the slowest contribution (typically with a decay time constant $>0.5 \mathrm{~ns}$ ).

\section{Solid-state fluorescence of 1a-d}

In contrast to AcPyr, which is almost non-emissive in the solid state (it exhibits extremely weak fluorescence centred at 486 $\mathrm{nm}$ ), ketones 1a-d are moderate solid-state emitters (Fig. 7 and 8 and Table 2). The solid state fluorescence of these compounds is centred at $530-567 \mathrm{~nm}$, i.e. $\sim 100 \mathrm{~nm}$ red-shifted compared to solution emission. This large shift suggests that the emissive state may be rather different to that of the molecular monomer in solution. The fluorescence quantum yields are in the range of 0.04 (1b)-0.13 (1a). Compounds $1 \mathbf{b}$ and 1d did not show a noticeable increase in solid-state fluorescence quantum yields

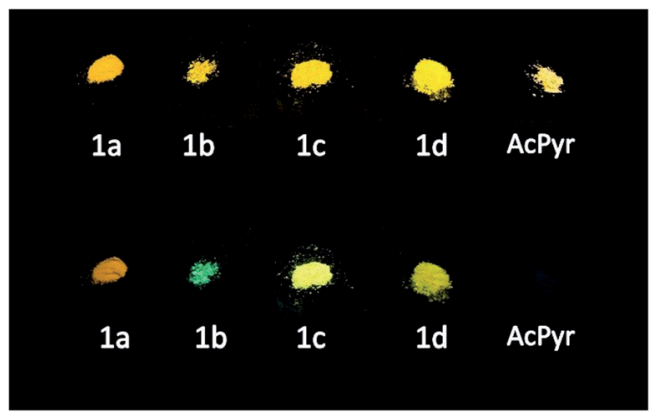

Fig. 7 Photographs of $1 a-d$ and AcPyr in the solid state under visible light (top row) und under $254 \mathrm{~nm}$ UV light (bottom row) illumination. as compared to the $\mathrm{CHCl}_{3}$ solution values, whereas 1a showed a 6 -fold and 1c a 2.5-fold increase of the quantum yield. Moreover, solid-state excitation spectra were also very much redshifted as compared to the $\mathrm{CHCl}_{3}$ solution, with $\lambda_{\max }$ of the first band in the $430-470 \mathrm{~nm}$ range, corresponding to red-shift values from $20 \mathrm{~nm}$ (1a) up to $55 \mathrm{~nm}(\mathbf{1 d})$.

Fluorescence time-resolved experiments of 1a were also performed in the solid state under ambient atmosphere. Fluorescence decay curves monitored at three different emission wavelengths $\left(\lambda_{\mathrm{em}}=540,590\right.$ and $\left.640 \mathrm{~nm}\right)$ are shown in Fig. 9.

Global analysis was successfully applied to the three decays with multiexponential fitting and revealed three common components: two long decay times, $\tau_{1}=13.2 \mathrm{~ns}$ and $\tau_{2}=27.4 \mathrm{~ns}$

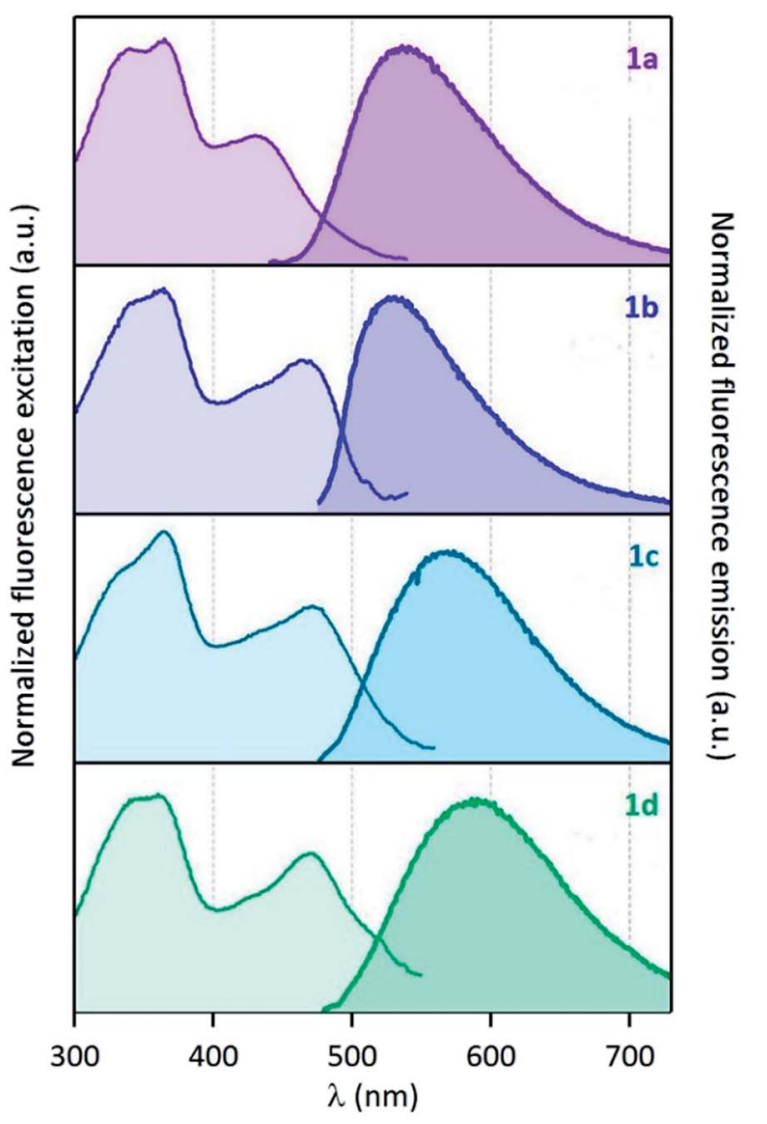

Fig. 8 (Left) normalized excitation and (right) emission spectra of compounds $1 \mathrm{a}-\mathrm{d}$ recorded in the solid state (powder inserted in an integration sphere). $\lambda_{\text {excit }}=430 \mathrm{~nm}$ for $1 \mathrm{a}$ and $460 \mathrm{~nm}$ for $1 \mathrm{~b}-\mathrm{d}$. 


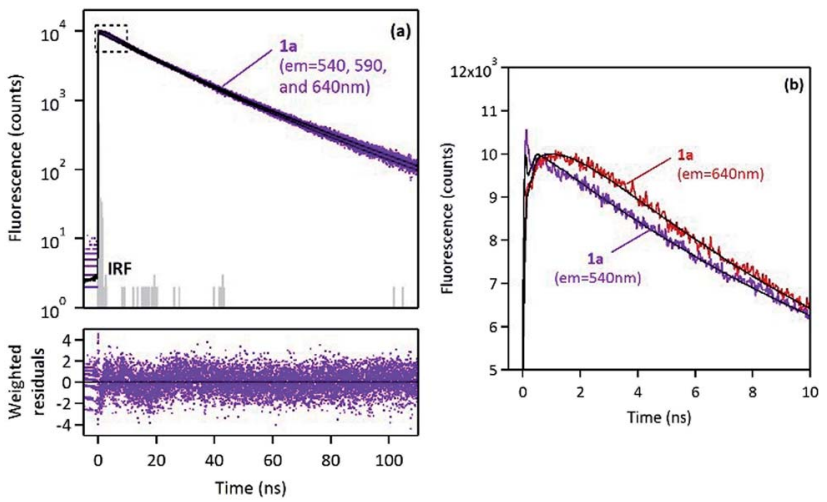

Fig. 9 (a) Fluorescence decay curves of $1 a$ in the solid state, monitored at three different emission wavelengths $\left(\lambda_{\mathrm{em}}=540,590\right.$ and 640 $\mathrm{nm}$ ), with multiexponential fitting and weighted residuals. (b) Zoomed region at short times, where excimer-type rise-time is clearly observed at $\lambda_{\mathrm{em}}=640 \mathrm{~nm}$, but absent at $\lambda_{\mathrm{em}}=540 \mathrm{~nm}$.

(which represented more than $99 \%$ of the fraction of intensity) and a short component, $\tau_{3} \sim 0.6-1.2 \mathrm{~ns}$, which appeared either as a decay time (for $\lambda_{\mathrm{em}}=540 \mathrm{~nm}$ ) or as a rise-time (for $\lambda_{\mathrm{em}}=$ 590 and $640 \mathrm{~nm}$ ), as shown in Fig. 9. Such a short decay time in the blue-edge of the spectrum corresponding to a short risetime in the red part of the spectrum is a typical signature of excimer formation which occurs within the crystal with a fast kinetic rate. This mechanism is rather consistent with the faceto-face orientation of the pyrene moieties in the crystal lattice, as revealed by X-ray diffraction (vide supra).

It is generally believed that such an aggregation leads to nonemissive species (H-aggregates). However, some examples of emissive H-aggregates have been reported. ${ }^{32-34}$ In such aggregates, emission may arise from restriction of intramolecular rotation (RIR), blocking of nonradiative decay channels and from the formation of emissive solid-state excimers (meaning not only "excited dimers" but also "excited oligomers"). Consequently, 1a-d emitted in the solid-state as monomers (a green emission), but they also interacted very quickly in the excited state and led to strongly emissive excimer species (a red emission), and then following a very slow decay rate. This situation is different from the one observed in solution, where only fast monomer decays were observed.

It should be emphasized that solid-state fluorescence, which is essential for various industrial applications, is still a relatively rare phenomenon because of the ubiquitous aggregationcaused quenching (ACQ) effect. Solid-state fluorophores exhibiting the aggregation-induced emission enhancement (AIEE) effect were discovered only very recently. ${ }^{35-37}$ Moreover, such fluorophores bearing a flat $\pi$-conjugated pyrene moiety are relatively rare. ${ }^{38-40}$ Strong yellow solid-state emission of 1a-d, with long fluorescence decays, makes them promising candidates for the design of luminescent materials and devices.

\section{Comparative DFT and TD DFTstudy of 1d and AcPyr}

To gain deeper insight into the influence of an acetylenic bond on the electronic structure of pyrene carbonyl chromophore
DFT and TD DFT calculations were performed on the simplest ynone (1d) and AcPyr. It has to be mentioned that a DFT study of the latter molecule was recently published by Konishi et al. ${ }^{15}$ However, in the present work we focused our interest on a comparison of both compounds at the same calculation level. The B3PW91 functional with a 6-311+g(d) basis set was used since it provided satisfying results for structurally close alkynoylferrocenes. ${ }^{41}$

First we scanned the potential energy $v s$. the dihedral angle between the pyrenyl moiety and the CO group (approximated by the C2-C1-C17-O1 angle), for 1d and AcPyr (Fig. 10).

The most stable geometry of $\mathbf{1 d}$ is planar with the carbonyl oxygen interacting with $\mathrm{C} 3-\mathrm{H}$ (Fig. 10a and c). The same conformation is present in the crystals of 1a (vide supra), a small twist of the CO group being attributable to the packing effects.

It is more stable by $2.34 \mathrm{kcal} \mathrm{mol}^{-1}$ than the conformation with the carbonyl oxygen directed towards $\mathrm{C} 14-\mathrm{H}$ and having the $C 2-\mathrm{C} 1-\mathrm{C} 17-\mathrm{O} 1$ dihedral angle $150^{\circ}$. This means that the equilibrium amount of the latter conformer at room temperature is $<3 \%$. The calculated barrier to rotation of the carbonyl group is $5.03 \mathrm{kcal} \mathrm{mol}^{-1}$. On the other hand, the most stable conformation of AcPyr (Fig. 10b and d) is nonplanar with a C2C1-C17-O1 dihedral angle $25^{\circ}$ and with the carbonyl oxygen also interacting with $\mathrm{C} 3-\mathrm{H}$ (the corresponding planar conformation is slightly less stable by $0.18 \mathrm{kcal} \mathrm{mol}^{-1}$ ). However, in this case the conformation with the dihedral $\mathrm{CO} /$ pyrene angle equal to $145^{\circ}$ is less stable only by $1.49 \mathrm{kcal} \mathrm{mol}^{-1}$, which means that the equilibrium amount of this conformation at room temperature may reach $8-10 \%$. The energy barrier to rotation of the acetyl group was found to be equal to $2.72 \mathrm{kcal} \mathrm{mol}^{-1}$. It is also worth noting that the pyrenyl-CO bond in 1d is significantly shorter than the analogous bond in AcPyr (1.483 and 1.495 $\AA$, respectively). This reveals a more efficient pyrenyl-CO conjugation in 1d, which is in line with the higher energy barrier for rotation around this bond in this compound than in AcPyr.
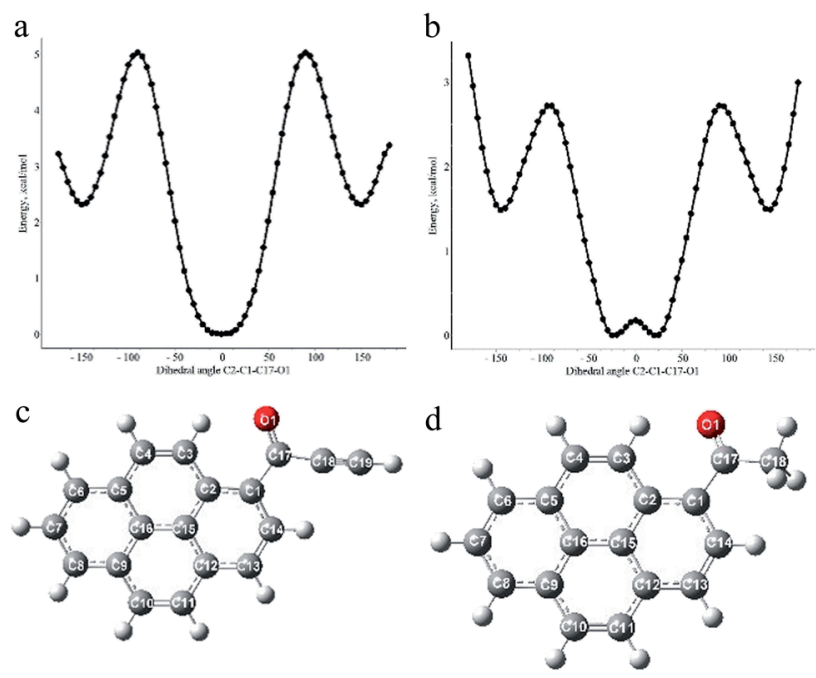

Fig. 10 Plots of calculated energy vs. C2-C1-C17-O1 dihedral angle for $1 \mathrm{~d}$ (a) and AcPyr (b) and optimized geometries of the most stable conformations of these compounds (c) and (d). 


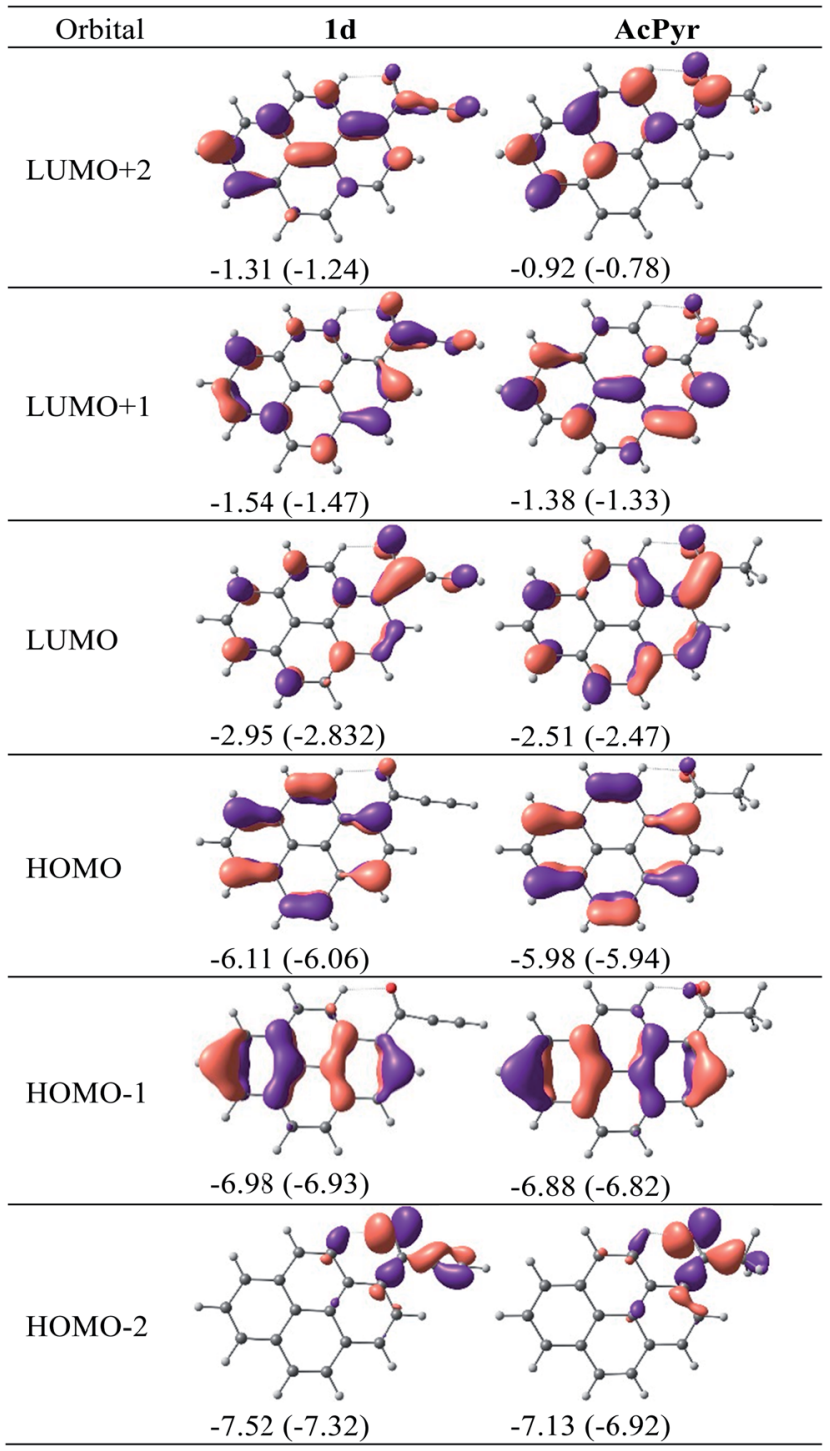

Fig. 11 DFT-calculated molecular orbitals for 1d and AcPyr. Orbital energies in $\mathrm{eV}$ in vacuum and (in parentheses) in $\mathrm{CHCl}_{3}$.

The calculated molecular orbitals of 1d and AcPyr are shown in Fig. 11

The HOMO-1 and HOMO of 1d and AcPyr differ only slightly in energy $(\sim 0.1 \mathrm{eV})$, presumably because in these orbitals the electron density is localized mostly at the pyrene moiety. In contrast, the HOMO-2 orbitals of these compounds are localized on the acyl groups, which results in a stronger $(0.4 \mathrm{eV})$ stabilization of this orbital in 1d. A significant difference in localization of electron density is observed for unoccupied LUMO, LUMO +1 and LUMO + 2 orbitals. In the case of AcPyr these orbitals are localized on the pyrenyl moiety and the carbonyl group, whereas delocalization is observed on the ethynyl group in the case of 1d. The strongest stabilization $(\sim 0.4-0.5 \mathrm{eV})$ is observed for the LUMO and LUMO +2 orbital of 1d, in comparison to the same orbital in AcPyr.

We have also calculated the electronic absorption spectra of 1d and AcPyr for isolated molecules and for a chloroform
Table 3 TD DFT-calculated electronic transitions in 1d and AcPyr

\begin{tabular}{lcll}
\hline Compound & \multicolumn{1}{l}{$\lambda_{\max } / \mathrm{nm}(\mathrm{eV})$} & $f$ & Main contribution \\
\hline \multicolumn{2}{l}{ (a) Isolated molecules } \\
1d & $407.4(3.04)$ & 0.3985 & $\mathrm{H} \rightarrow \mathrm{L}(0.95)$ \\
& $356.1(3.48)$ & 0.0527 & $\mathrm{H}-1 \rightarrow \mathrm{L}(0.79)$ \\
& $292.1(4.24)$ & 0.1297 & $\mathrm{H} \rightarrow \mathrm{L}+1(0.71)$ \\
AcPyr & $261.9(4.73)$ & 0.1231 & $\mathrm{H}-4 \rightarrow \mathrm{L}(0.79)$ \\
& $377.0(3.29)$ & 0.3523 & $\mathrm{H} \rightarrow \mathrm{L}(0.92)$ \\
& $283.5(4.37)$ & 0.1909 & $\mathrm{H} \rightarrow \mathrm{L}+1(0.45)$ \\
& & & $\mathrm{H}-1 \rightarrow \mathrm{L}(0.19)$ \\
& $247.6(5.01)$ & 0.1743 & $\mathrm{H}-4 \rightarrow \mathrm{L}(0.62)$
\end{tabular}

(b) $\mathrm{CHCl}_{3}$ solution

$\begin{array}{llll}\text { 1d } & 429.3(2.89) & 0.5538 & \mathrm{H} \rightarrow \mathrm{L}(0.97) \\ & 362.3(3.42) & 0.0774 & \mathrm{H}-1 \rightarrow \mathrm{L}(0.85) \\ 296.4(4.18) & 0.2104 & \mathrm{H} \rightarrow \mathrm{L}+1(0.70) \\ & 291.7(4.25) & 0.0510 & \mathrm{H} \rightarrow \mathrm{L}+2(0.73) \\ & 266.9(4.64) & 0.1416 & \mathrm{H}-4 \rightarrow \mathrm{L}(0.84) \\ & 249.9(4.96) & 0.1003 & \mathrm{H}-5 \rightarrow \mathrm{L}(0.89) \\ \text { AcPyr } & 386.8(3.21) & 0.4962 & \mathrm{H} \rightarrow \mathrm{L}(0.95) \\ & 298.7(4.15) & 0.0525 & \mathrm{H}-3 \rightarrow \mathrm{L}(0.64) \\ & & & \mathrm{H} \rightarrow \mathrm{L}+1(0.22) \\ & 287.0(4.32) & 0.2435 & \mathrm{H} \rightarrow \mathrm{L}+1(0.37) \\ & & & \mathrm{H}-3 \rightarrow \mathrm{L}(0.32)\end{array}$

solution using time-dependent DFT and a polarizable continuum model (PCM) using integral equation formalism variant (IEFPCM). The data are gathered in Table 3.

The lowest energy band may practically be considered a pure HOMO-LUMO transition for both compounds. The calculated wavelengths of this band for the chloroform solution are in good agreement with the experimental values (429 vs. $415 \mathrm{~nm}$ for $\mathbf{1 d}$ and 387 vs. $391 \mathrm{~nm}$ for AcPyr). It is generally believed that face-to-face $\mathrm{H}$-aggregates are non-emissive in terms of Kasha's theory of exciton coupling. However, some examples of emissive $\mathrm{H}$-aggregates have been reported..$^{32-34}$ In such aggregates, emission may arise from restriction of intramolecular rotation (RIR), blocking nonradiative decay channels or formation of emissive solid-state excimers (meaning not only "excited dimers" but also "excited oligomers"). A time-resolved fluorescence study clearly demonstrated that solid-state emission of $\mathbf{1 a}$ originated from dynamic excimers formed during and shortly after the laser pulse and emitting at longer wavelengths than monomeric molecules. Some contribution from the RIR effect is also possible since the intermolecular hydrogen bond network along with $\pi$-stacking may severely inhibit rotational motions in individual fluorophores in the crystals.

\section{Conclusions}

We elaborated an efficient method of Friedel-Crafts acylation of pyrene with conjugated alkynoic acids which led to a new class of pyrenyl fluorophores - 1-pyrenyl ynones. Comparison of the electronic absorption and fluorescence emission spectra of these compounds with those of the simplest saturated pyrenyl ketone, 1-acetylpyrene, revealed some of their advantages. The lowest energy absorption bands of 1-pyrenyl ynones were 
shifted bathochromically to the visible region, therefore these compounds can be conveniently excited with violet light. They exhibit more intense fluorescence than 1-acetylpyrene in a medium polarity environment. Their fluorescence maxima are shifted towards lower energies by 50-60 $\mathrm{nm}$ and their fluorescence lifetimes are substantially longer than those of 1-acetylpyrene. In contrast to the latter compound, they exhibit strong fluorescence in the solid state, which is associated with long decay times and which can be attributed to an efficient formation of excimer species. Finally, the simplest ynone, 1(pyren-1-yl)prop-2-yn-1-one, may be used in azide-alkyne click chemistry for introduction of a fluorescent pyrenyl tag to diverse molecular structures (biomolecules, polymers, etc.) This possibility is currently being studied in our laboratory and the results will be published in due time.

\section{Acknowledgements}

Financial support from the National Science Centre (Grant Harmonia UMO-2012/04/M/ST5/00712) is gratefully acknowledged. This research was also supported in part by the PL-Grid Infrastructure.

\section{Notes and references}

1 T. M. Figueira-Duarte and K. Müllen, Chem. Rev., 2011, 111, 7260-7314.

2 F. Ciardelli, G. Ruggeri and A. Pucci, Chem. Soc. Rev., 2013, 42, 857-870.

3 A. M. Breul, M. D. Hager and U. S. Schubert, Chem. Soc. Rev., 2013, 42, 5366-5407.

4 G. Drummen, Molecules, 2012, 17, 14067-14090.

5 G. Bains, A. B. Patel and V. Narayanaswami, Molecules, 2011, 16, 7909-7935.

6 R. Métivier, I. Leray, M. Roy-Auberger, N. Zanier-Szydlowski and B. Valeur, New J. Chem., 2002, 26, 411-415.

7 R. Métivier, I. Leray, J.-P. Lefevre, M. Roy-Auberger, N. ZanierSzydlowski and B. Valeur, Phys. Chem. Chem. Phys., 2003, 5, 758-766.

8 A. Bencini and V. Lippolis, Coord. Chem. Rev., 2012, 256, 149169.

9 J. Xie, M. Ménand, S. Maisonneuve and R. Métivier, J. Org. Chem., 2007, 72, 5980-5985.

10 L. Zöphel, V. Enkelmann and K. Müllen, Org. Lett., 2013, 15, 804-807.

11 X. Feng, J.-Y. Hu, F. Iwanaga, N. Seto, C. Redshaw, M. R. J. Elsegood and T. Yamato, Org. Lett., 2013, 15, 13181321.

12 Y. Niko, S. Kawauchi, S. Otsu, K. Tokumaru and G.-i. Konishi, J. Org. Chem., 2013, 78, 3196-3207.

13 M. Ottonelli, M. Piccardo, D. Duce, S. Thea and G. Dellepiane, J. Phys. Chem. A, 2012, 116, 611-630.

14 C. X. Yao, H. B. Kraatz and R. P. Steer, Photochem. Photobiol. Sci., 2005, 4, 191-199.
15 Y. Niko, Y. Hiroshige, S. Kawauchi and G.-i. Konishi, Tetrahedron, 2012, 68, 6177-6185.

16 Y. Niko, Y. Hiroshige, S. Kawauchi and G. I. Konishi, J. Org. Chem., 2012, 77, 3986-3996.

17 Y. Niko, S. Kawauchi and G. I. Konishi, Tetrahedron Lett., 2011, 52, 4843-4847.

18 A. Jana, S. Atta, S. K. Sarkar and N. D. P. Singh, Tetrahedron, 2010, 66, 9798-9807.

19 K. Szczubiałka, Ł. Moczek, A. Goliszek, M. Nowakowska, A. Kotzev and A. Laschewsky, J. Fluorine Chem., 2005, 126, 1409-1418.

20 L. Bucsiova, P. Hrdlovič and 亡̌. Chmela, J. Photochem. Photobiol., A, 2001, 143, 59-68.

21 Y. Niko and G.-i. Konishi, J. Synth. Org. Chem Jpn., 2012, 70, 918-927.

22 D. A. Fleming, C. J. Thode and M. E. Williams, Chem. Mater., 2006, 18, 2327-2334.

23 S. P. Sau and P. J. Hrdlicka, J. Org. Chem., 2011, 77, 5-16.

24 D. Plażuk and J. Zakrzewski, J. Organomet. Chem., 2009, 694, 1802-1806.

25 B. Willy and T. J. J. Müller, ARKIVOC, 2008, i, 195-208.

26 M. Navidi and B. Movassagh, Monatsh. Chem., 2013, 144, 1363-1367.

27 W. Sun, Y. Wang, X. Wu and X. Yao, Green Chem., 2013, 15, 2356-2360.

28 B. Huang, L. Yin and M. Cai, New J. Chem., 2013, 37, 31373144 .

29 M. Navidi, B. Movassagh and S. Rayati, Appl. Catal., A, 2013, 452, 24-28.

30 N. M. Carballeira, Chem. Phys. Lipids, 2013, 172-173, 58-66.

31 U. Koch and P. L. A. Popelier, J. Phys. Chem., 1995, 99, 97479754.

32 V. Karunakaran, D. D. Prabhu and S. Das, J. Phys. Chem. C, 2013, 117, 9404-9415.

33 U. Rösch, S. Yao, R. Wortmann and F. Würthner, Angew. Chem., Int. Ed., 2006, 45, 7026-7030.

34 S. Varghese and S. Das, J. Phys. Chem. Lett., 2011, 2, 863-873. 35 S. P. Anthony, ChemPlusChem, 2012, 77, 518-531.

36 M. Shimizu and T. Hiyama, Chem.-Asian J., 2010, 5, 15161531.

37 Y. Hong, J. W. Y. Lam and B. Z. Tang, Chem. Commun., 2009, 4332-4353.

38 Q. Feng, M. Wang, B. Dong, C. Xu, J. Zhao and H. Zhang, CrystEngComm, 2013, 15, 3623-3629.

39 P. Kotchapradist, N. Prachumrak, R. Tarsang, S. Jungsuttiwong, T. Keawin, T. Sudyoadsuk and V. Promarak, J. Mater. Chem. C, 2013, 1, 4916-4924.

40 Y. Li, D. Wang, L. Wang, Z. Li, Q. Cui, H. Zhang and H. Yang, J. Lumin., 2012, 132, 1010-1014.

41 D. Plażuk, J. Zakrzewski, K. Nakatani, A. Makal, K. Woźniak and S. Domagala, RSC Adv., 2012, 2, 3512-3524. 